\title{
Aprendizaje-servicio en tiempos de pandemia: campaña contra el dengue a través de Juegotecas Virtuales destinadas a niñas y niños en aislamiento
}

\author{
Marcela Martínez Vivot \\ Graciela Paolicchi \\ Lucía Abreu \\ Francina Basso \\ Universidad de Buenos Aires, Argentina
}

\section{Resumen}

La actual situación a raíz de la pandemia del COVID-19 implicó adecuaciones y modificaciones en las prácticas que afectan a la enseñanza. Esta experiencia se desarrolló en momentos de aislamiento social preventivo y obligatorio en Argentina durante el período marzo-julio del 2020. El objetivo de esta fue potenciar en los estudiantes -dentro del marco de un proyecto de extensión universitario interdisciplinario, donde se aplica el aprendizaje-servicio- competencias genéricas de responsabilidad social, motivación, creatividad, trabajo en equipo y la aplicación de teoría y práctica, llevado a la modalidad del espacio virtual. Asimismo, se consideró fomentar en ellos la responsabilidad social en las buenas prácticas de prevención del COVID-19 y de dengue. Se trabajó utilizando los siguientes lemas: Quédate en casa; Cuídate; y Sin mosquito no hay Dengue. Para evaluar la experiencia se utilizaron diferentes instrumentos cualitativos y en todos los casos revelaron que se cumplieron los objetivos propuestos, destacándose una alta satisfacción por participar en la experiencia virtual tanto de los voluntarios, como los niños y sus familias.

\section{Palabras clave}

Aprendizaje-servicio, COVID-19, virtualidad, niños, juego.

Fecha de recepción: 24/VIII/2020

Fecha de aceptación: 6/XI/2020 aislamiento. RIDAS, Revista Iberoamericana de Aprendizaje Servicio, 10, 12-22. 


\title{
Service-learning in times of a pandemic: campaign against dengue fever through virtual games for children in isolation
}

\begin{abstract}
The current social and health situation amidst the COVID-19 pandemic necessarily entails adjustments and changes in educational practices. The experience this paper relates took place at a time of preventive and compulsory social isolation in Argentina between March and July 2020. The experience was developed within the framework of a university interdisciplinary outreach project, based on service-learning, and it aimed at developing students' generic competences, such as social responsibility, motivation, creativity, teamwork and the application of theory and practice, all within a virtual space as a consequence of the current context caused by the pandemic. The experience also aimed to foster students' social responsibility with good practices for the prevention of COVID-19 and dengue fever. To evaluate the experience, different qualitative instruments were used, and, in all cases, they revealed that the proposed objectives were met, highlighting a high level of satisfaction with the virtual experience, not only among the student volunteers, but also among the children and their families. Virtual platforms enabled us to recreate face-to-face work without losing the university social responsibility, and they also helped to promote responsibility from childhood, motivating everyone to face the needs imposed by the current reality as a team.
\end{abstract}

\section{Keywords}

Service-learning, COVID-19, virtuality, children, game. aislamiento. RIDAS, Revista Iberoamericana de Aprendizaje Servicio, 10, 12-22. 


\section{Introducción}

Esta experiencia se desarrolló en el contexto de la situación sanitaria mundial a raíz del COVID-19. Desde el día 20 de marzo, en Argentina, comenzó a regir el aislamiento social preventivo y obligatorio. Desde el proyecto de extensión interdisciplinario de voluntariado subsidiado por la Universidad de Buenos Aires (UBA) sostenemos que este aislamiento traerá consecuencias psíquicas y emocionales en los niños con quienes trabajamos. Asimismo, se ha observado de qué manera dicha situación ha provocado intensas manifestaciones en el entramado familiar que acompaña a ese niño, siendo necesario intervenir ofreciendo otros recursos de acompañamiento, con la intención de provocar mayor bienestar en dicho contexto.

En primera instancia, destacamos que surgieron los siguientes interrogantes: ¿De qué manera se podía implementar la metodología de aprendizaje-servicio en los proyectos que estábamos realizando de manera presencial?; ¿Cómo se podía motivar a los voluntarios para que sus aprendizajes tengan impacto en la resolución de problemas reales como el dengue?; ¿Cómo se podía incluir a la comunidad general para resolver este problema y potenciar la responsabilidad social?; ¿De qué manera llevar adelante el trabajo de Juegotecas Barriales en este período de aislamiento?

En segunda instancia, y con la intención de ofrecer respuestas a los interrogantes planteados, se ha considerado la adaptación del trabajo a las plataformas virtuales como Zoom, WhatsApp, Instagram Live, videos online, entre otras. Si bien las características de esta nueva modalidad virtual difieren notablemente del trabajo en campo, se considera que han permitido sostener la continuidad del trabajo en equipo, como así también la responsabilidad social universitaria, favoreciendo la transmisión a las familias y niños.

Retomando esto último y mencionando brevemente lo que respecta a la campaña de prevención y concientización del dengue, durante este periodo, se han establecido encuentros virtuales junto a todo el equipo de trabajo con el objetivo de repensar la transmisión de dicha campaña y de qué manera hacer partícipe de ello a la propia comunidad. Por otro lado, en lo que respecta al programa de las Juegotecas Barriales, fue "aggiornado" a encuentros virtuales y ha sido denominada Juegotecas Virtuales. Dicha propuesta es llevada a cabo por los estudiantes del programa y tiene como principal objetivo ofrecer un momento de juego y recreación para los niños que se encuentran en aislamiento.

\section{Objetivos y metodología}

Todo proyecto de aprendizaje-servicio que se precie de calidad debe tener doble intencionalidad, pedagógica y de servicio. Por lo tanto, los objetivos de esta experiencia se refieren tanto al servicio comunitario como a los aprendizajes del alumnado. aislamiento. RIDAS, Revista Iberoamericana de Aprendizaje Servicio, 10, 12-22. 
- Objetivos referidos a la dimensión social: están enfocados en la familia y niños de la comunidad general que están atravesando el aislamiento social obligatorio por el COVID-19. Por ello, el objetivo de servicio fue fomentar la adquisición de la responsabilidad social en las buenas prácticas de prevención del COVID-19 y el dengue en niños mediante su participación activa con dispositivos tecnológicos. Éstos últimos permitieron traspasar la virtualidad y a partir de una consigna brindada, los niños se expresaron verbalmente y utilizaron juguetes acordes a la edad, para acompañar los significados de la prevención.

\section{- Objetivos referidos a la} dimensión pedagógica: están enfocados en los aprendizajes de los estudiantes voluntarios que participan activamente en el proyecto interdisciplinario socio comunitario universitario UBANEX y de Juegotecas Barriales y que no pueden hacerlo de forma presencial debido al aislamiento. Entonces, el objetivo de aprendizaje fue potenciar en el alumnado, las competencias genéricas de responsabilidad social, motivación, creatividad, trabajo en equipo interdisciplinario y la aplicación de teoría y práctica para que elaboren un instrumento lúdico que pueda viralizarse, para niños que están atravesando el aislamiento social.

En lo que respecta a la población de referencia, en primera instancia fueron 77 los estudiantes universitarios voluntarios que participaban presencialmente en los proyectos socio comunitarios de Extensión de la UBA. EI criterio de selección de la muestra para esta experiencia, realizado en una segunda instancia, fue a través de las reuniones virtuales de reflexión del grupo de voluntarios, donde continuaron 47 del total referido. Con respecto a las titulaciones de pertenencia del proyecto, fueron estudiantes de Ciencias Veterinarias, Diseño, Comunicación Social y Psicología de la UBA. Dichos estudiantes mostraron particular interés en participar del proyecto bajo esta nueva modalidad virtual, logrando ocupar un rol activo y participativo en lo que refiere al cumplimiento de los objetivos de trabajo planteados.

La muestra de niños estuvo constituida por 53, siendo los del programa como así también participaron activamente los hijos, sobrinos y nietos de los voluntarios de ambos proyectos. Para la realización del video se contó con el consentimiento informado de las madres y los padres para poder difundirlo. Esta experiencia de aprendizaje-servicio se presentó en las escuelas de cada uno de los niños que participaron activamente de los videos; el Campus virtual de la Cátedra de enfermedades Infecciosas de la Facultad de Ciencias Veterinarias y de la Cátedra de Evolutiva Niñez II de la Facultad de Psicología; las redes sociales (Instagram y Facebook) institucionales como también de los voluntarios, amigos y familiares de los niños que participaron de la experiencia.

Por último, se detallan a continuación las herramientas metodológicas utilizadas dado que son los medios para obtener información válida y confiable proporcionada por las unidades aislamiento. RIDAS, Revista Iberoamericana de Aprendizaje Servicio, 10, 12-22. 
informantes. Asimismo, la meta de todo investigador es la de establecer rapport con los informantes, concepto difícil de definir pero que según Taylor y Bogdan (1992) se relaciona con "comunicar la simpatía que se siente por los informantes y lograr que ellos la acepten como sincera" (p.55). Las técnicas de recogida de información con sus instrumentos son una cuestión clave en todo proceso de investigación. El tema de las evidencias es fundamental porque considera que son ellas las que decidirán finalmente si las explicaciones de la investigación pueden ser consideradas válidas o no (Borsotti, 2007).

En esta experiencia se ha optado por estrategias de recogida de información cualitativas, a saber: 1) entrevistas y autoevaluación, utilizando como instrumento un cuestionario (según escala Likert); 2) análisis de documentación de material producido (videos) por los voluntarios sobre las buenas prácticas de prevención del COVID-19 y el dengue protagonizado por los niños; 3) la observación (directa, indirecta, controlada y participante) utilizando como instrumento las notas de campo.

En este sentido, estas estrategias nos permiten obtener información exhaustiva y variada sobre las actitudes, los valores, las creencias, las opiniones, etc. de las personas entrevistadas. Con estas estrategias se pretende acceder al punto de vista de los actores, para conocer así cómo ellos interpretan sus experiencias en sus propios términos.

\section{Marco teórico}

Una de las funciones sustantivas de la Universidad consiste en formar ciudadanos participativos y motivados, los cuales se encuentren provistos de un sentido crítico y capaces de analizar los problemas de la sociedad, buscar soluciones para los que se planteen a la sociedad, aplicar éstas y asumir responsabilidades sociales (Organización de las Naciones Unidas para la Educación, la Ciencia y la Cultura [UNESCO], 1998). En este sentido, Tapia (2017) afirma que, "en algunos contextos el concepto de responsabilidad social universitaria se emparenta con la posibilidad de 'devolver' a la sociedad el sostén social que hace posible la existencia misma de la universidad pública" (p.162). Justamente el aprendizajeservicio resulta una metodología interesante en la cual los estudiantes aprenden realizando un servicio a la sociedad.

Sin embargo, estudiantes y profesores voluntarios, que estaban habituados a trabajar de manera presencial, han tenido que afrontar esta inesperada situación de pandemia. La enseñanza a distancia exige el rediseño integral de la misma (Basabe, 2020; Feldman, 2020; Soletic 2020) e impone repensar el diseño curricular y su transmisión.

En esta instancia de adaptación a la virtualidad se destacó la observación como método de investigación. En la medida que aporta una explicación detallada de todo el proceso es en donde se pueden incluir ciertos presupuestos, entre ellos las coordenadas espacio-temporales;

Martínez Vivot, M., Paolicchi, G., Abreu, L. y Basso, F. (2020). Aprendizaje-servicio en tiempos de pandemia: campaña contra el dengue a través de Juegotecas Virtuales destinadas a niñas y niños en aislamiento. RIDAS, Revista Iberoamericana de Aprendizaje Servicio, 10, 12-22.

DOI10.1344/RIDAS2020.10.2 
dónde se sitúa, cuándo y cómo se desarrolla la acción; la postura de las personas que trabajan en el proyecto; la percepción de conflictos manifiestos o latentes dentro de la comunidad, o de la comunidad con el exterior; el ambiente del servicio; la relación de las participantes con el profesorado; la distribución del espacio, abundancia o carencia de medios, ornamentación y las sugerencias recibidas sobre el desarrollo de la evaluación (Folgueiras, 2005; Folgueiras, Luna y Palou, 2010). Puede ser utilizada como un elemento más de análisis y de rigor de la propia investigación, y es considerada como una herramienta útil, puesto que aporta información sobre el proceso de investigación (Monistrol, 2007).

Justamente, en la experiencia del Proyecto de Extensión universitaria se destacó la observación como estrategia básica donde el investigador se implique y participe activamente en la situación para obtener una comprensión de ésta en profundidad (Basabe, 2020). De esta forma, los estudiantes voluntarios han podido realizar observación participante con niños, incorporando también la participación de sus hijos y sobrinos, quienes se encontraron imposibilitados de asistir a las instituciones escolares como consecuencia de la pandemia.

Por lo tanto, que los niños no estén asistiendo a la escuela los pone en un grupo vulnerable, no es el tema de la tarea o el aprendizaje; es decir, no ponemos énfasis en las cuestiones específicas del proceso de enseñanzaaprendizaje (algo que consideramos sumamente relevante) sino que el trabajo del Proyecto de Extensión universitaria ha sido observar los efectos que esta situación podría ocasionar al detectar reacciones de angustia, regresiones respecto de logros alcanzados, miedos y agresividad. Se les interrumpió la sociabilización. La falta de interacciones con pares, compartir espacios de juegos, actividades deportivas y salidas recreativas ha generado ciertos conflictos que, de no ser tratados, podrían derivar en problemáticas que por su seriedad implicarían tener que acudir a otro tipo de intervenciones. Paolicchi (en Hartmann, 2020) explica que la vulnerabilidad se produce porque hay una suerte de retroceso:
El niño ya salía, ya tenía sus amigos, y otra vez se ve todo el día en situaciones que ya había superado. En los peores casos, encima, donde había maltrato o abuso infantil, todo se perpetúa porque no están disponibles los espacios donde esto saltaba a la vista (párr. 18 y 19).

Al respecto, Bendel (en Hartmann, 2020) ratificó lo expuesto "[...]bajó la cantidad de llamados denunciando violencia, lo que confirma el dato de que ocho de cada diez violencias ocurren intramuros y son detectadas por la escuela, el hospital, el club. Pero al no estar esos lugares, no se denuncia" (párr.20); pero este tema excede el espacio del presente artículo y queda pendiente para un futuro.

\section{Descripción de la experiencia}

Cuando se trabaja con estudiantes como participantes activos de un proyecto donde se brindan soluciones a

Martínez Vivot, M., Paolicchi, G., Abreu, L. y Basso, F. (2020). Aprendizaje-servicio en tiempos de pandemia: campaña contra el dengue a través de Juegotecas Virtuales destinadas a niñas y niños en aislamiento. RIDAS, Revista Iberoamericana de Aprendizaje Servicio, 10, 12-22.

DOI10.1344/RIDAS2020.10.2 
problemas concretos, resulta sumamente motivador observar cómo los conocimientos de los voluntarios estudiantes se comparten e interactúan con la comunidad, inquietada por circunstancias reales, en nuestro caso frente a la enfermedad del Dengue.

Mientras la atención -tanto social, económica como de intervenciones sanitarias- continúa haciendo foco sobre el COVID-19, es menester informar que las personas contagiadas por dengue en Argentina suman 41.754 en el período comprendido entre julio 2019 hasta mayo de 2020, superando a los registrados en el año 2015-2016 en el mismo período, que hasta el momento era el que contaba con el mayor número de infectados con 41.402. Si la medición se toma por año calendario, en las primeras 19 semanas de 2020 se registraron 41.349 casos contra los 40.463 registrados en 2016 . Así lo informó el Boletín Integrado de Vigilancia que elabora el Ministerio de Salud de la Nación. Estos datos provocan en nosotros, como agentes de acciones sociales y comunitarias, la necesidad de continuar y fortalecer nuestra acción directamente dirigida a la problemática mencionada. Asimismo, en este período tuvimos casos de dengue entre los graduados voluntarios, hecho que sumó mucha preocupación y angustia que se cristalizó en la necesidad de continuar con las actividades de prevención que veníamos realizando presencialmente, pero de manera virtual. Y también, añadir las buenas prácticas de prevención del COVID-19 de cara a esta pandemia ya que, sin lugar a duda, el aislamiento social constituye un grave problema de salud pública.

En consecuencia, vale mencionar la encuesta realizada por el Instituto de Neurociencia Cognitiva y Traslacional de la Universidad Favaloro (Universidades hoy, 2020) a más de 10.000 personas, la cual reveló que alrededor de un tercio de los encuestados atravesaba un estado depresivo o ansioso significativo. Además, según los expertos, se estima que al ir extendiéndose la cuarentena también irá en aumento la cantidad de personas con esta sintomatología. Ante esta problemática se tuvo una reunión por la plataforma Zoom entre los voluntarios alumnos y graduados para repensar las actividades desde lo virtual, en la cual algunos de ellos plantearon la necesidad de encontrar la manera de continuar con aquellos objetivos del proyecto UBANEX que pudieran realizarse a distancia. Se priorizaron a niños menores de 7 años por los efectos negativos del aislamiento social ya que psicólogos y pediatras advierten sobre los riesgos para la salud mental y emocional. Se consensuó entonces pensar el aislamiento social como un proceso que va a tener etapas y que en cada una iremos recuperando espacios que parecían perdidos. El cambio de rutinas establecido, la falta de contacto afectivo con los grupos de pares, la suspensión de actividades recreativas en espacios públicos y el aislamiento en el contexto familiar más íntimo, trae aparejado una sensación de malestar e incomodidad en cada una de las personas. En este punto es fundamental que tanto los adultos como los niños perciban que esa suspensión de sus vidas no será eterna, provocando así la necesidad de

Martínez Vivot, M., Paolicchi, G., Abreu, L. y Basso, F. (2020). Aprendizaje-servicio en tiempos de pandemia: campaña contra el dengue a través de Juegotecas Virtuales destinadas a niñas y niños en aislamiento. RIDAS, Revista Iberoamericana de Aprendizaje Servicio, 10, 12-22.

DOI 10.1344/RIDAS2020.10.2 
motivarlos a dirigir su atención a nuevos intereses y/o nuevas rutinas mediadas por el contexto actual.

Se cree que fomentar la participación y responsabilidad social desde la pequeña edad a través del juego ha sido una prioridad. Asimismo, y para que estas actividades puedan replicarse en la comunidad, se pensó en desafiar a otros proyectos de voluntariado a otras instituciones educativas y a otros niños con sus familias a que realicen actividades similares a distancia. Se comenzó a distribuir en las redes, apenas comenzó la cuarentena, como una propuesta lúdica, para replicar diferentes acciones relacionadas con canto, interpretaciones y música, entre otras, a distancia.

Los dos temas relevantes que tratamos en esta experiencia se basaron en las buenas prácticas de prevención del COVID-19 y del dengue. Primeramente, se presentaron como disparadores, dos videos ${ }^{1}$ con una duración de sólo 1 minuto, para que sea accesible a distribuir por celular y que además permita sostener la atención de los niños. El primero, relacionado con la prevención del COVID-19 era de libre distribución del Gobierno Nacional. El segundo, fue realizado por los estudiantes voluntarios del proyecto con dibujos de animación, presentando de manera sencilla, con vocabulario acorde para que los más pequeños entiendan todo lo relacionado con la prevención y transmisión del dengue.

\footnotetext{
1 Videos campaña contra el dengue, realizados por el equipo de extensión universitaria: https://drive.google.com/drive/u/1/folders/1WX p7WyLwriFwV-siL202P bgPOB98sd1
}

De esta manera los estudiantes potenciaban diversas competencias (búsqueda de información, creatividad, capacidad de síntesis, trabajo interdisciplinario, aplicación de la teoría en la práctica y la capacidad de explicar en términos sencillos el lenguaje científico).

Posteriormente, se pidió a los niños que actuaran como investigadores, exploradores o detectives en sus propias casas para buscar, junto a su familia, elementos donde pueda anidar el mosquito (como botellas, latas, neumáticos, juguetes, trozos de plástico y lona, bidones cortados, portamacetas, bebederos y cualquier otro receptáculo), y lo muestren en un video filmado por su madre o padre, de manera que se involucraban en un proyecto que proponía asumir la responsabilidad familiar. Asimismo, para la temática de prevención del COVID-19 estos pequeños investigadores expusieron en otro video los presupuestos relacionados con la prevención de este, ejercitando la responsabilidad infantil en construcción.

Finalmente, se recopiló cada uno de los videos de los investigadores como así también aquellas frases espontáneas relacionadas al período de cuarentena y cómo prevenir el COVID-19. Todo este material se editó en dos videos y se presentó en un panel de cátedra para que los estudiantes universitarios realizaran articulaciones teóricoprácticas y ejercitaran sobre el aprendizaje-servicio. Además, se distribuyeron virtualmente en las Juegotecas Barriales, jardines de infantes y las escuelas que trabajan intensamente a distancia y ya estaban

Martínez Vivot, M., Paolicchi, G., Abreu, L. y Basso, F. (2020). Aprendizaje-servicio en tiempos de pandemia: campaña contra el dengue a través de Juegotecas Virtuales destinadas a niñas y niños en aislamiento. RIDAS, Revista Iberoamericana de Aprendizaje Servicio, 10, 12-22.

DOI 10.1344/RIDAS2020.10.2 
ávidos con esta modalidad para que los niños participen activamente traspasando la virtualidad. Esta instancia de transmisión tanto a la comunidad educativa como al ámbito social y familiar representa una valiosa herramienta de trabajo en donde el aprendizaje y el servicio se hacen presentes de manera innegable.

La recogida de información se realizó una vez finalizada cada actividad y luego de las reuniones de reflexión a distancia. Vale destacar que cada niño realizaba una actividad separada de los otros niños ya sea en diferente tiempo como espacio, y que luego se editaban todos los videos juntos. Asimismo, las entrevistas y la autoevaluación, así como el análisis del material de video producido, se realizó una vez finalizadas todas las actividades.

La autoevaluación, diseñada por el equipo, fue destinada a los estudiantes involucrados en el nuevo formato de Juegotecas Virtuales; y consistió en ponderar según escala Likert del 1 al 5 (1 Nada satisfecho / 5= Totalmente satisfecho) y responder brevemente 3 preguntas: (1) ¿Cómo te sentís en esta nueva experiencia de Juegotecas Virtuales?; (2) ¿Qué tan difícil fue para vos implementar los juegos en modo virtual?; y (3) ¿Qué tan importante consideras la adaptación de las Juegotecas a modo virtual en consecuencia de la pandemia? Con relación a las preguntas 1 y 3 , la totalidad ponderó con 5; mientras que la pregunta 2 obtuvo un punto menos (puntaje de 4) y esto, se adjudica como parte de la complejidad del pasaje de una modalidad presencial-grupal en la cual los estudiantes tenían experiencia previa, a una modalidad virtual, que implica sostener una experiencia novedosa y de forma individual. Sin embargo, al momento del desarrollo de las Juegotecas Virtuales, los niños mostraron naturalidad incorporando al estudiante -mediante el celular 0 computadora- a su juego. Aquí, un fragmento de una nota de campo realizada por una de las estudiantes que ejemplifica con claridad la naturalidad con la que una niña maneja el espacio virtual:

"L. (5 años) tomó el celular y me dijo 'vamos a ver a los gatitos' $y$ empezó a correr (dirigiéndose a hacia su habitación), cuando llegamos se le cayó el celular, 'lo siento' me dijo, como si yo también me hubiera caído!".

Consideramos que se pudo mantener la propuesta gracias a la excelente predisposición de los niños a jugar virtualmente y al acompañamiento de sus familias.

\section{Conclusiones}

Considerando el contexto que atraviesa a toda la sociedad, es importante destacar que durante este período se ha podido llevar adelante un gran trabajo de equipo, promoviendo hábitos saludables de cuidado y prevención de la salud, tanto física como psíquica.

El trabajo de prevención del dengue a través de las Juegotecas Virtuales ha permitido sostener el contacto con la comunidad y las familias, atendiendo a las necesidades propias del contexto actual como así también promoviendo los objetivos de trabajo de cada

Martínez Vivot, M., Paolicchi, G., Abreu, L. y Basso, F. (2020). Aprendizaje-servicio en tiempos de pandemia: campaña contra el dengue a través de Juegotecas Virtuales destinadas a niñas y niños en aislamiento. RIDAS, Revista Iberoamericana de Aprendizaje Servicio, 10, 12-22.

DOI 10.1344/RIDAS2020.10.2 
proyecto respectivamente fortaleciendo la construcción de conocimiento colaborativo. Asimismo, se considera una experiencia fructífera en tanto, a través de las plataformas digitales, se pudo "aggiornar" el trabajo sin perder el vínculo con los niños. Se ha podido redefinir la modalidad de trabajo sin perder la responsabilidad social universitaria, saliendo de nuestra zona de confort y enfrentando en equipo las necesidades que impone la realidad actual. Se cree que el entramado entre estudiantes, docentes y profesionales de las distintas áreas de estudio implicadas posibilitó fortalecer el vínculo desde una mirada multidisciplinaria y ampliada al contexto.

Se sostuvo el trabajo de prevención en pos de la salud para disminuir el impacto del aislamiento, a raíz del COVID-19, en niños; es decir, que se tuvo una mirada atenta a la problemática actual, considerando los desafíos que implica a nivel social, económico y de salud mental. Además, en relación específica a la prevención del dengue, se mantuvo la responsabilidad de manera anual y eso beneficia a la población en el sentido que ya está educada para las épocas de mayor contagio. En esta prevención comunitaria, nuestro equipo multidisciplinario fue -y continúa siendo- agentes.

Asimismo, reflexionando parte de lo desarrollado anteriormente, es importante mencionar aquí los efectos psicológicos producidos en los niños y sus familias como consecuencia del aislamiento social. En este punto, cabe ponderar la importancia del acompañamiento y sostén realizado a cada una de las familias durante este período, ofreciendo herramientas que se orientan hacia lo terapéutico generado por el trabajo del juego y facilitando interacciones familiares $y$ sociales en procura de un mayor bienestar. Como profesionales agentes de salud en el ámbito social, hemos puesto como prioridad fortalecer el vínculo entre el trabajo de los proyectos mencionados y la población a la que se dirige, con el objetivo de disminuir los efectos producto por esta pandemia mundial.

Por último, y como cierre de este primer cuatrimestre virtual, el equipo de profesores, docentes, investigadores y estudiantes realizaron un video compilado recitando el poema "La esperanza" de Alexis Valdés ${ }^{2}$. Entendemos que es una forma de fortalecer la trama académica como así también la social.

\section{Referencias bibliográficas}

Basabe, L. (2020). Alternativas para la evaluación de los aprendizajes en formatos no presenciales. Serie "Enseñanza sin presencialidad: reflexiones y orientaciones pedagógicas". Documento 3. Buenos Aires, Argentina: CITEP, UBA.

Borsotti, C. (2007). Temas de metodología de la investigación en ciencias sociales empíricas. Madrid, España: Mino y Dávila Editores.

\footnotetext{
${ }^{2}$ https://www.facebook.com/watch/?v=1806576 039480306
}

Martínez Vivot, M., Paolicchi, G., Abreu, L. y Basso, F. (2020). Aprendizaje-servicio en tiempos de pandemia: campaña contra el dengue a través de Juegotecas Virtuales destinadas a niñas y niños en aislamiento. RIDAS, Revista Iberoamericana de Aprendizaje Servicio, 10, 12-22. DOI 10.1344/RIDAS2020.10.2 
Especialistas advierten "epidemia" de estrés postraumático por la cuarentena. (12 de mayo de 2020). Universidades HOY. Recuperado de:

http://universidadeshoy.com/despacho s.asp?cod des $=69853$ \&id seccion $=233$

Feldman, D. (2020). Enseñanza sin presencialidad: algunas notas para una situación no esperada. Serie

"Enseñanza sin presencialidad: reflexiones y orientaciones pedagógicas". Documento 1. Buenos Aires, Argentina: CITEP, UBA.

Folgueiras, P. (2005). De la tolerancia al reconocimiento: programa de formación para una ciudadanía (tesis doctoral). Recuperado de: https://www.tdx.cat/handle/10803/235 $\underline{1 \# \text { page }=1}$

Folgueiras, P., Luna, E. y Palou, B. (2010). Ciudadanía, participación y aprendizaje-servicio: del centro educativo a la comunidad. Recuperado de:

https://www.academia.edu/17549200/ Ciudadan\%C3\%ADa aprendizaje y ser vicio del centro educativo a la comu nidad

Hartmann, I. (20 de abril de 2020). Coronavirus en Argentina: ¿Es hora de que los chicos salgan al aire libre? Clarín. Recuperado de:

https://www.clarin.com/sociedad/coron avirus-argentina-hora-chicos-salganaire-libre- 0 GPsNb-9C-.html

Monistrol, O. (2007). El trabajo de campo en investigación cualitativa (II). Nure Investigación, (29). Recuperado de:
https://www.nureinvestigacion.es/OJS/i ndex.php/nure/article/view/350/341

Soletic, A. (2020). Recomendaciones para el diseño de la enseñanza en la virtualidad. Serie "Enseñanza sin presencialidad: reflexiones y orientaciones pedagógicas". Documento 2. Buenos Aires, Argentina: CITEP, UBA.

Organización de las Naciones Unidas para la Educación, la Ciencia y la Cultura [UNESCO]. (1998). Declaración mundial sobre la educación superior en el siglo XXI: visión y acción .

Recuperado de:

https://www.oei.es/historico/salactsi/D ECLARACION MUNDIAL EDUCACION S UPERIOR.pdf

Taylor, S.J. y Bogdan, R. (1992). Introducción a los métodos cualitativos de investigación. Barcelona, España:

Paidós.

Tapia, M. N. (2017). Aprendizaje Servicio Solidario. El sistema educativo y las organizaciones juveniles. Buenos Aires, Argentina: Ciudad Nueva.

Martínez Vivot, M., Paolicchi, G., Abreu, L. y Basso, F. (2020). Aprendizaje-servicio en tiempos de pandemia: campaña contra el dengue a través de Juegotecas Virtuales destinadas a niñas y niños en aislamiento. RIDAS, Revista Iberoamericana de Aprendizaje Servicio, 10, 12-22. DOI 10.1344/RIDAS2020.10.2 